\title{
Analysis tools for non-radially pulsating objects
}

\author{
D. J. Wright, K. R. Pollard, P. L. Cottrell
}

Department of Physics and Astronomy, University of Canterbury, Christchurch, New Zealand

\begin{abstract}
At the University of Canterbury we have been developing a set of tools for the analysis of spectra of varying types of non-radially pulsating objects. This set currently includes: calculation of the moments, calculations of the phase across the profile as well as basic binary profile fitting for determination of orbital characteristics and projected rotational velocity $(v \sin i)$ measurement. Recently the ability to calculate cross-correlation profiles using either specified or synthesized line lists has been added, all implemented in MATLAB. A number of observations of $\gamma$ Doradus candidates is currently being used to test these tools. For information on our observing facilities see Pollard et al. (2007).
\end{abstract}

\section{Introduction}

The set of tools that is being developed for the analysis of spectra of different types of non-radially pulsating objects include computing the moments of line profiles (Balona 1986), calculations of the phase across the profile (Telting \& Schrijvers 1997), binary profile fitting for the determination of orbital characteristics and projected rotational velocity $(v \sin i)$ measurements. We will employ profile inversions on fast rotators in the near future.

Objects of this type require very high signal-to-noise $(\mathrm{S} / \mathrm{N})$ spectra for precise measurements of the line profiles. This need is lessened by the use of a cross-correlation technique to obtain a representative line profile. A number of observations of $\gamma$ Doradus candidates and $\delta$ Scuti stars is currently being used to test these tools.

\section{Cross-correlation and projected rotational velocity}

Using cross-correlation of an object's spectrum with a template of Delta Functions shifted to the rest wavelength position of the line and scaled to their relative depths, we achieve a high $\mathrm{S} / \mathrm{N}$ representation of the line profile. This is valid for lines similarly distorted by the pulsation.

The projected rotational velocity of the object is measured using the Fourier analysis technique outlined by Gray (1992, see his Fig. $17.12(\mathrm{a})$ ). The position of the first minimum on the abscissa of a Fourier-transformed line profile is compared with a theoretically calculated position obtained by the Fourier transform of the convolution of the spectrograph's instrumental profile with the rotational broadening function. This theoretical position has been tested and is not very sensitive to the instrumental width.

\section{Line profile variations and periodicity of the moments}

Line profiles are tested for variation by examining the residuals after subtraction of the average line profile. If enough observations are obtained the moments of the line profiles are tested for variability. We can examine the periodicities present in the various moments to obtain some basic pulsation mode information. For example, the periodogram of the first moment shows all periodicities present in the star (with cross terms), whilst the second moment does 
not show the axisymmetric modes $(m=0)$ that are potentially present. The line moments technique is useful for $\delta$ Scuti stars where the main line profile variations are significant. However, for $\gamma$ Doradus stars, where the line profile variations are not so apparent, other techniques have greater sensitivity and are therefore more useful for this class of non-radial pulsator. An example of this is the technique of line profile inversion (Berdyugina 1998).

Acknowledgments. We thank the Royal Society of New Zealand (Canterbury Branch) for support toward travel to this conference and the Physics and Astronomy Department at the University of Canterbury.

\section{References}

Balona L. A., 1986, MNRAS, 220, 647

Berdyugina S. V., 1998, A\&A, 338, 97

Gray D. F., 1992, The Observation and Analysis of Stellar Photospheres, Cambridge University Press Pollard K. R., Wright D. J., Cottrell P. L., et al., 2007, Comm. Asteroseis., 150, 133

Telting J., Schrijvers C., 1997, A\&A, 317, 723

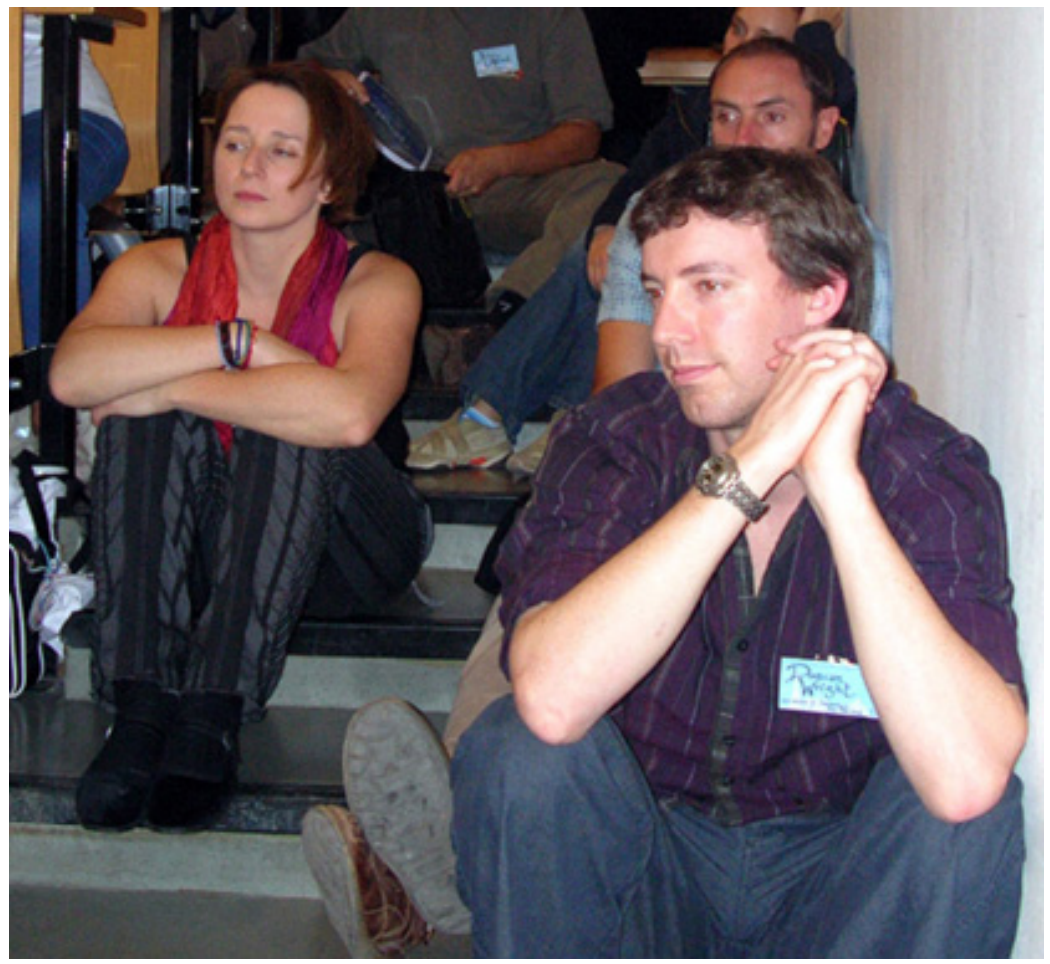

Theresa Lüftinger and Duncan Wright listening to an obviously interesting talk. Partially occulted in the background: Thierry Morel. 